- $\quad$ Supporting Information -

\title{
UV-Spectroscopy of Peptide and Protein Polyanions in vacuo: Signature of the Ionization State of Tyrosine
}

Laure Joly, Rodolphe Antoine, Abdul-Rahman Allouche, Michel Broyer, Jérôme Lemoine, Philippe Dugourd

Université de Lyon, Université Lyon 1; CNRS; ${ }^{\dagger}$ LASIM UMR 5579, bât. A. Kastler ; Sciences Analytiques, UMR 5180, 43 Bvd. du 11 Novembre 1918, 69622 Villeurbanne cedex, France

Table 1 : Intrinsic pKa values of ionizable groups found in proteins.

in T. E. Creighton, Proteins : Structures and Molecular Properties. W. H. Freeman; 2nd edition, New York (1992)

\begin{tabular}{cc}
\hline Ionizable Group & Observed pKa \\
\hline$\alpha$-carboxyl & $3.5-4.3$ \\
$\beta$-carboxyl (Asp) & $3.9-4.0$ \\
$\gamma$-carboxyl (Glu) & $4.3-4.5$ \\
thiol (Cys) & $9.0-9.5$ \\
phenolic hydroxyl (Tyr) & $10.0-10.3$ \\
$\beta$-hydroxyl (Ser, Thr) & 13 \\
\hline
\end{tabular}

A chain

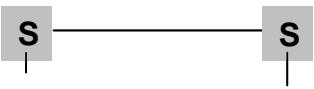

Gly-lle-Val-Glu-GIn-Cys-Cys-Ala-Ser-Val-Cys-Ser-Leu-Tyr-GIn-Leu-Glu-Asn-Tyr-Cys-Asn

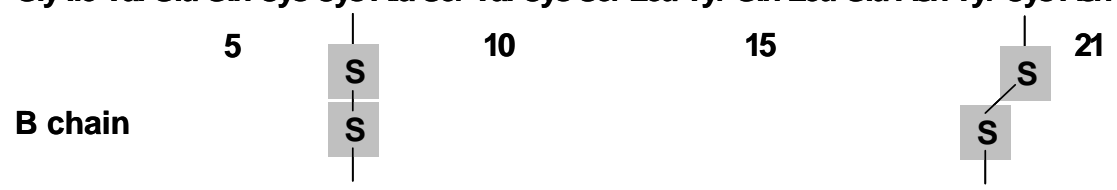

Phe-Val-Asn-GIn-His-Leu-Cys-Gly-Ser-His-Leu-Val-Glu-Ala-Leu-Tyr-Leu-Val-Cys-Gly-Glu-Arg-Gly-Phe-Phe-Tyr-Thr-Pro-Lys-Ala

5

10

15

20

25

30

Figure 1S: Amino acid sequence of bovine insulin (molecular weight 5733.5 Da) 
a)

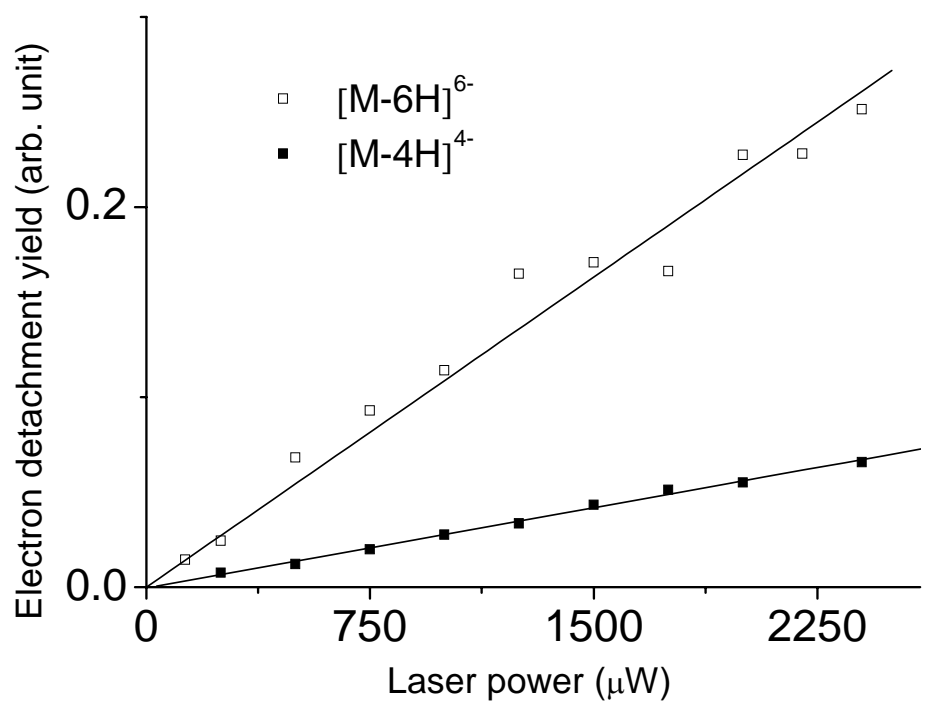

b)

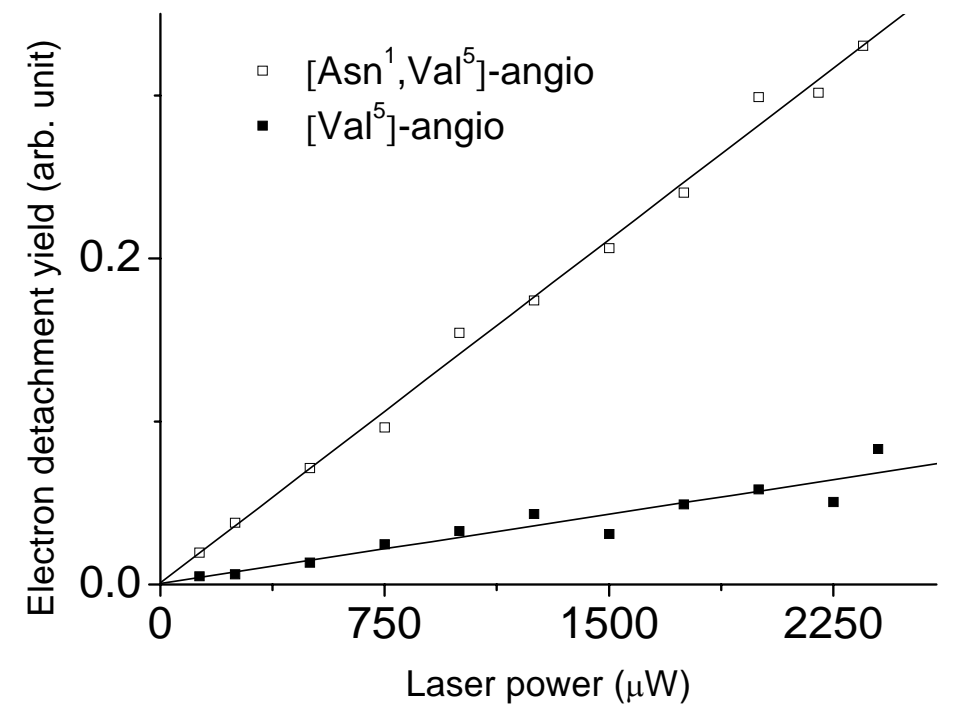

Figure 2S : Electron detachment yield (In((parent + oxidized product)/parent) ) measured as a function of the laser power at $\lambda=260 \mathrm{~nm}$ for the two variants of angiotensin II (a) and two charge states of insulin (b). The laser power was controlled using a half wave plate and a polarizer. Solid lines are linear fits of the electron detachment yields. 


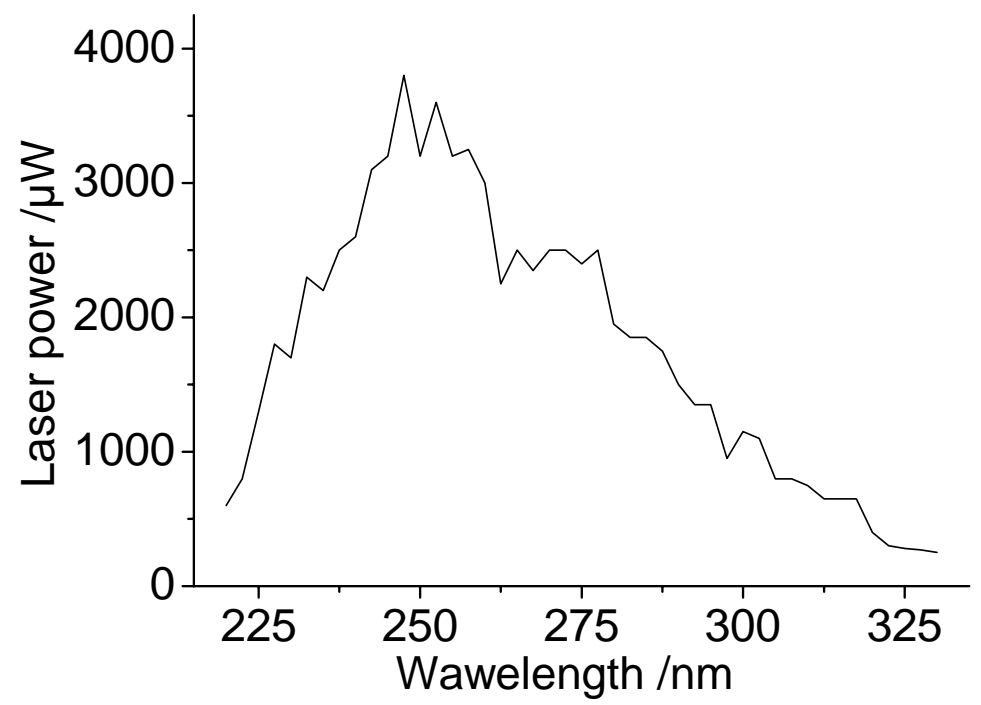

Figure 3S : Dependence of the laser power at the entrance of the trap as a function of the wavelength. 

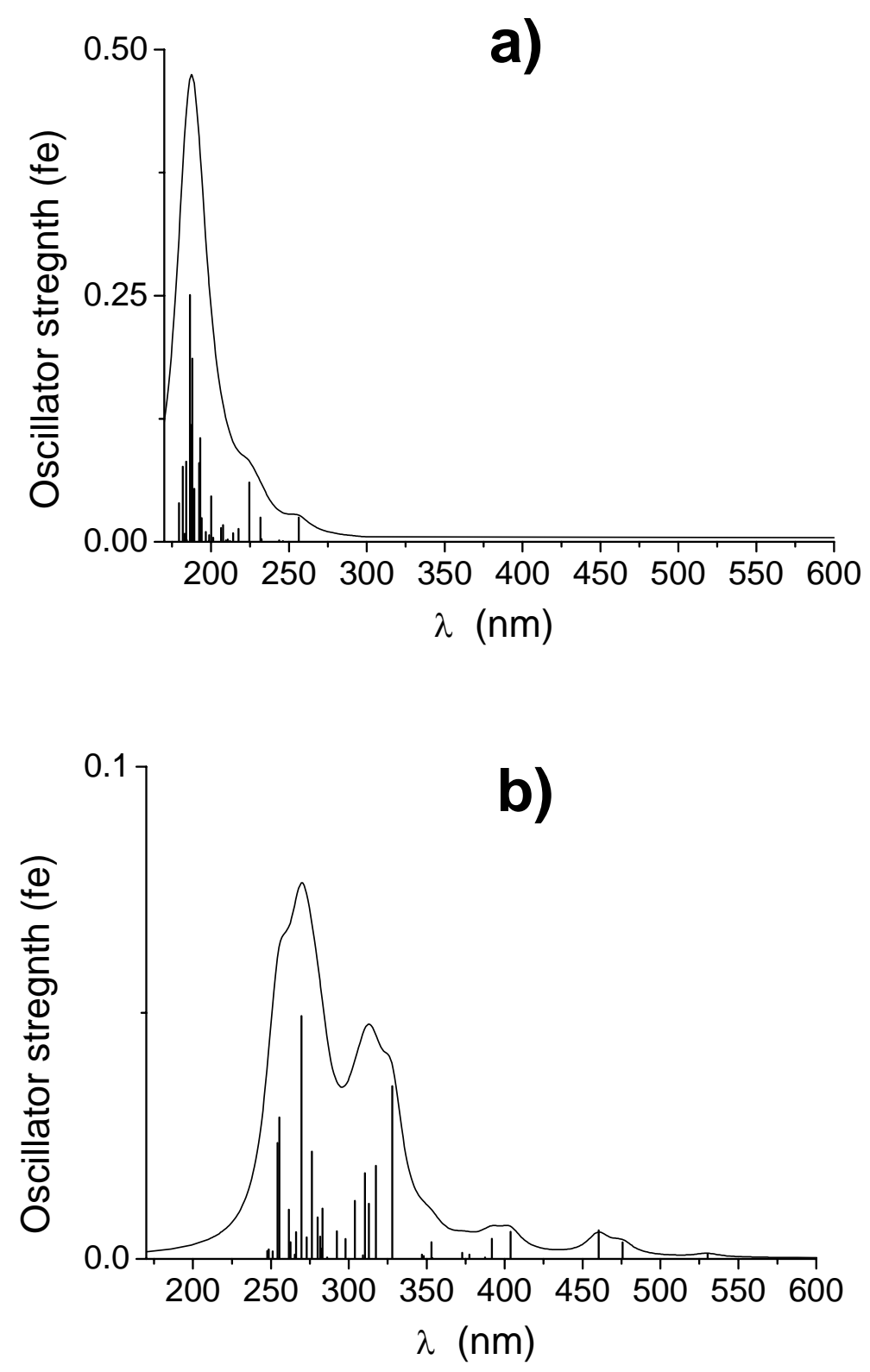

Figure 4S : TD-DFT calculation of the optically allowed transitions $\lambda$ (in nanometers) and oscillator strengths (fe) for the neutral (a) and ionized form (tyrosinate) (b) of tyrosine. Structural properties of the neutral and the tyrosinate forms of tyrosine amino-acid have been determined using density functional theory (DFT) with the B3LYP hybrid functional and the aug-cc-pvdz basis set. Absorption spectra were obtained from time dependent density functional method (TD-DFT) using B3LYP with the aug-cc-pvdz basis set.The number of transitions (singlet states) included in the calculation is 30 for the neutral form and 40 for the ionized form of tyrosine. Broadening of the lines is simulated by Lorentzian functions with a width of $9 \mathrm{~nm}$.

In figure $4 \mathrm{~S} \mathrm{~b}$ ), the transition at $269 \mathrm{~nm}(\mathrm{fe}=0.049)$ is mainly due to a $\pi-\pi^{*}$ excitation of the carbonyl bond of the tyrosyl side chain. The transition at $327 \mathrm{~nm}(\mathrm{fe}=0.033)$ is mainly due to a $\pi-\pi^{*}$ excitation of the benzyl ring. 